\title{
Visit Entropy Associated with Hospital Readmission Rates
}

\author{
Gregory M. Garrison, MD, MS, Rachel Keuseman, MD, Buck Bania, MD, \\ Paul Robelia, MD, and Jennifer Pecina, MD
}

Purpose: The chronic disease model suggests continuity of care and team-based care can improve outcomes for multimorbidity patients and reduce hospitalizations. Continuity of care following admission has had mixed effects on readmission rates; however, its effect before admission has not been well studied. Increased outpatient care organization and continuity before admission is hypothesized to reduce the odds of readmission.

Methods: In a cohort of 14,662 primary care patients from a Patient-Centered Medical Home (PCMH) practice, continuity of care in the 12 months before admission was assessed using 3 established metrics; usual provider continuity (UPC), dispersion continuity of care (COC), and sequence continuity (SECON). In addition, because these established metrics may not accurately reflect continuity in planned teambased care, a new metric called visit entropy (VE) was used to quantify the disorganization of visits. Multivariate logistic regression was performed to examine the relationship between readmission within 30 days and continuity while controlling for known readmission risk factors abstracted from an electronic medical record.

Results: Higher VE was associated with readmission (odds ratio, 1.10; 95\% confidence interval, 1.02 to 1.19). The continuity measures of UPC, COC, and SECON were not associated with readmission.

Conclusions: Disorganized medical care, characterized by a higher VE, is associated with higher odds of readmission among hospitalized primary care patients. An association between traditional measures of continuity (UPC, COC, and SECON) and readmission was not found. (J Am Board Fam Med 2017;30:63-70.)

Keywords: Ambulatory Care, Chronic Disease, Continuity of Patient Care, Electronic Health Records, Entropy, Hospitalization, Logistic Models, Odds Ratio, Patient Readmission, Patient-centered Care, Primary Health Care, Risk Factors

Hospital readmission within 30 days of discharge is common, occurring in almost $20 \%$ of US Medicare patients and costing $\$ 17.4$ billion in 2004. ${ }^{1} \mathrm{Un}$ planned readmissions have often been considered a marker for poor quality inpatient care or ineffective hospital-to-home transitions. ${ }^{2-7}$ Various interventions using pharmacists and advance-practice

This article was externally peer reviewed.

Submitted 7 June 2016; revised 28 September 2016; accepted 3 October 2016.

From the Department of Family Medicine, Mayo Clinic, Rochester, MN

Funding: Department of Family Medicine, Mayo Clinic, Rochester, MN, Small Grant Funding, Mayo CCaTS grant UL1TR000135.

Conflict of interest: none declared.

Corresponding author: Gregory M. Garrison, MD, MS, 200 First Street SW, Rochester, MN 55905 (E-mail: garrison.gregory@mayo.edu). nurses have been shown to assist patients with the transition process and reduce readmissions. ${ }^{7-10} \mathrm{~A}$ patient centered medical home provides a natural foundation on which to support the transition of patients from hospital to outpatient care. ${ }^{11}$

Continuity of care has been defined by the Institute of Medicine as longitudinal care by a single health-care provider coupled with effective and timely communication of health information. ${ }^{12}$ The chronic disease model suggests continuity of care and team-based care are necessary to improve outcomes for multi-comorbidity patients and reduce hospitalizations. ${ }^{13-16}$ Conversion from a walk-in clinic to a medical-home clinic was shown to reduce early readmissions. ${ }^{17}$ However, increased continuity of care following hospital discharge has had mixed effects on readmissions. ${ }^{18,19}$ Likewise, 
continuity of care has had variable effects on initial hospitalizations. ${ }^{20-22}$ The effect of continuity of care before initial admission on the risk of subsequent readmission has not been well studied.

Jee and Cabana $^{23}$ have classified measures of continuity of care into 5 broad categories that measure density of provider visits, dispersion of provider visits, sequence of provider visits, duration of provider relationships, and subjective patient estimates of continuity. Of these, the first 3 categories can be represented by mathematical formulae that attempt to derive a quantifiable and objective measure of continuity of care. The Usual Provider Continuity (UPC), a measure of density defined by Breslau and Reeb ${ }^{24}$ in 1975 as the fraction of visits made to the most frequent or primary physician, is commonly used in the literature to quantify continuity. ${ }^{23-26}$ Sequence and dispersion of provider visits are represented by the Sequence Continuity (SECON) and Continuity of Care (COC) indices respectively. UPC, SECON, and COC indices suffer from skewed distributions, division-by-zero problems, and the inability to distinguish planned referrals from random visits. ${ }^{27}$ Thus, they may be less valid in today's health care environment with an emphasis on team-based care. Visit Entropy (VE) represents a new continuity of care index that solves many of these potential shortcomings by measuring the disorganization of an outpatient visit pattern. ${ }^{27}$ It is based on well-established theory regarding entropy in physics and information science. ${ }^{27-29}$ Patients who visit multiple different clinicians have a more disorganized visit pattern and hence higher VE than those who visit a limited set of clinicians frequently.

We hypothesize increased outpatient care organization and continuity in the 12 months before index hospitalization reduces the odds of 30-day readmission after controlling for demographic, utilization, and medical complexity factors. Because there is little agreement on an accepted measure of continuity of care, we have chosen to analyze the 4 measures of continuity of care (UPC, SECON, COC, VE) separately. ${ }^{23,25,27,30-32}$

\section{Methods \\ cobort}

A dataset of all hospitalizations of adult primary care patients at our institution during 2011 to 2013 was utilized for this study. Only patients giving consent for retrospective chart review research were included in the dataset. All patients were local community members and had an identified primary care physician at 1 of 5 primary care clinical sites located in and around Rochester, Minnesota. Each primary care clinical site is a PCMH. Patients were excluded from analysis if they were discharged from a psychiatric unit or obstetric labor and delivery. In addition, a small number of patients enrolled in an intensive care transitions management program for frail high-risk elders were excluded (441 patients). There were 26,278 admissions for 14,662 unique patients. The first inpatient hospital admission of every patient during the study period was selected for analysis. Data regarding demographics, dates of hospitalizations and emergency department (ED) visits, length of stay, the Charlson Comorbidity Index ${ }^{33}$, and 12 months of previous outpatient visits were obtained from the electronic health record. The study was reviewed and approved by the Mayo Clinic Institutional Review Board.

\section{Continuity Measures}

Four different measures, UPC, COC, SECON, and VE were used to quantify continuity in the analysis. The 4 measures represent density of provider visits (UPC), dispersion of provider visits (COC), sequence of provider visits (SECON), and the disorganization of provider visits (VE). These 4 continuity measures were explained and contrasted in detail previously. ${ }^{27}$ The formula for computing each measure from a patient's outpatient visit pattern is shown in Tables 1. VE quantifies the disorganization of a patient's visit pattern to health care providers. VE decreases in value as the continuity of care increases. It is minimized when a patient has perfect continuity of care by only visiting their primary physician, and it is maximized when a patient visits several unique clinicians once each. To illustrate the 4 continuity measures, Table 2 shows their values for several different visit patterns. In contrast with the other measures, VE is able to distinguish planned care referrals (case F) from unplanned visits to differing subspecialists (case D). In addition, VE correctly quantifies that 4 visits to the primary care physician (case $\mathrm{H}$ ) has higher continuity than 1 or 2 visits to the primary physician (cases B and E). UPC, COC, and SECON all suffer from undefined division by zero when zero or 1 visits are observed (cases A and B). Although $\mathrm{VE}$ is defined even if no visits are observed, its scale 
Table 1. Formula Used to Calculate Continuity Measures

\begin{tabular}{|c|c|}
\hline Measure & Definition \\
\hline Density (UPC) & $\begin{array}{l}\max \left(\frac{n_{i}}{N}\right) \text { where } \mathrm{N}=\text { total visits } \\
\mathrm{n}_{i}=\text { number visits to } i^{t h} \text { provider }\end{array}$ \\
\hline Dispersion (COC) & $\begin{array}{l}\frac{\sum_{i=1}^{p}\left(n_{i}^{2}-N\right)}{N(N-1)} \text { where } \mathrm{N}=\text { total } \\
\quad \text { visits } \\
n_{i}=\text { total visits to } i^{t h} \text { provider } \\
\mathrm{p}=\text { total number of providers }\end{array}$ \\
\hline Sequence (SECON) & $\begin{array}{l}\sum_{j=1}^{N-1} c_{j} \\
\overline{N-1} \text { where } \mathrm{N}=\text { total visits } \\
C_{j}=\left\{\begin{array}{c}1 \text { if visit } j \text { and } j+1 \\
\text { are to same provider } \\
0 \text { otherwise }\end{array}\right.\end{array}$ \\
\hline $\begin{array}{l}\text { Entropy/Disorganization } \\
\text { (VE) }\end{array}$ & $\begin{array}{l}-\sum_{i=1}^{k} p\left(x_{i}\right) \log _{2} p\left(x_{i}\right) \\
\text { where } p\left(x_{i}\right)=\frac{n_{i}+1 / k}{N+1} \\
n_{i}=\text { the number of visits to the } \\
\mathrm{i}^{\text {th }} \text { provider } \\
k=\text { the total number of possible } \\
\text { providers } \\
N=\text { the total number of visits }\end{array}$ \\
\hline
\end{tabular}

COC, dispersion continuity of care; SECON, sequence continuity; UPC, usual provider continuity; VE, visit entropy.

depends on determining the maximum number of different potential providers. We assumed this to be the highest number of distinct providers visited by any patient in the year preceding admission $(\mathrm{k}=$ 62).

\section{Analysis}

The dependent variable was readmission or death within 30 days of hospital discharge. The 30-day period matches the Centers for Medicare and Med- icaid Services definition of hospital readmission. Although methods to adjust for potentially avoidable readmissions exist, ${ }^{34}$ we choose to consider any readmission to eliminate subjectivity. Independent variables include age, sex, marital status, Charlson comorbidity score, number of prior hospitalizations, number of prior ED visits, and length of stay.

All data were abstracted electronically and analyzed using R version 3.02 (http://www.r-project. org/). Bivariate statistics for the various factors and the dependent variable readmission were computed using a Wilcoxon rank-sum test for numeric data and Fisher exact test or $\chi^{2}$ test for categorical data. $P<.05$ were considered significant. For each measure of continuity, a multivariate analysis using logistic regression was conducted to adjust for known readmission risk factors. ${ }^{1,35-43}$ Odds ratios with 95\% confidence intervals were calculated. Direct comparison of the 4 non-nested models over the entire dataset using Akaike's Information Criterion was not valid due to the fact that the UPC, COC, and SECON models all excluded different data due to their inherent division-by-zero problem.

\section{Results}

The first admission of 14,662 patients was analyzed, and $1697(11.6 \%)$ patients were readmitted within 30 days of discharge. Excluding ED visits on the day of admission, the median patient made 8 outpatient visits and saw 5 different health care providers in the 12 months preceding their admission. However, 473 (3.2\%) patients had no outpatient visits in the preceding 12 months.

Table 2. Value of Continuity Measures for Various Visit Patterns

\begin{tabular}{|c|c|c|c|c|}
\hline Visit Pattern & UPC & $\mathrm{COC}$ & SECON & $\mathrm{VE}$ \\
\hline A) No visits & Undefined & Undefined & Undefined & 4.14 \\
\hline B) 1 primary physician visit & 1.00 & Undefined & Undefined & 2.72 \\
\hline C) 1 primary physician visit, 1 ED visit, 1 dermatology visit, 1 Urgent care visit & 0.25 & 0.00 & 0.00 & 2.37 \\
\hline $\begin{array}{l}\text { D) } 1 \text { primary physician visit, } 1 \text { cardiology visit, } 1 \text { primary physician visit, } 1 \\
\text { cardiology visit (different specialist) }\end{array}$ & 0.50 & 0.17 & 0.33 & 2.11 \\
\hline E) 2 primary physician visits & 1.00 & 1.00 & 1.00 & 1.99 \\
\hline $\begin{array}{l}\text { F) } 1 \text { primary physician visit, } 1 \text { cardiology visit, } 1 \text { primary physician visit, } 1 \\
\text { cardiology visit (same referral) }\end{array}$ & 0.50 & 0.33 & 0.00 & 1.85 \\
\hline $\begin{array}{l}\text { G) } 2 \text { nurse practitioner visits, } 1 \text { primary physician visit, } 1 \text { nurse practitioner } \\
\text { visit (same NP) }\end{array}$ & 0.50 & 0.17 & 0.33 & 1.74 \\
\hline H) 4 primary physician visits & 1.00 & 1.00 & 1.00 & 1.31 \\
\hline
\end{tabular}

COC, dispersion continuity of care; ED, emergency department; SECON, sequence continuity; UPC, usual provider continuity; VE, visit entropy. 
Table 3. Descriptive Statistics of Independent Variables for the Cohort by Group

\begin{tabular}{lccc}
\hline Characteristic & Not Readmitted & Readmitted & $P$-Value* \\
\hline $\mathrm{N}$ & 12,965 & 1697 & \\
Age, mean (SD) & $61.2(19.3)$ & $64.1(19.3)$ & \\
Sex & & $901(53.1 \%)$ & $<.001$ \\
$\quad$ Female (\%) & $7034(54.3 \%)$ & $796(46.9 \%)$ & \\
$\quad$ Male (\%) & $5931(45.7 \%)$ & $734(43.3 \%)$ & .379 \\
Marital status & & $963(56.7 \%)$ & \\
$\quad$ Not married (\%) & $5114(39.4 \%)$ & $4.9(3.7)$ & $<.7(6.2)$ \\
$\quad$ Married (\%) & $7851(60.6 \%)$ & $1.2(2.2)$ & $<.003$ \\
Carlson Comorbidity Index, mean (SD) & $3.3(3.3)$ & $0.3(1.0)$ & $<.001$ \\
Length of stay, mean (SD), days & $3.2(3.6)$ & $12.4(12.3)$ & $<.001$ \\
Previous 6 months ED Visits, mean (SD) & $0.8(1.6)$ & $7.7(6.5)$ & $<.001$ \\
Previous 12 months Hospitalizations, mean (SD) & $0.1(0.5)$ & $0.37(0.22)$ & $<.001$ \\
Previous 12 months Outpatient Visits, mean (SD) & $9.8(9.2)$ & $0.12(0.16)$ & $<.001$ \\
No. Distinct providers, 12 months, mean (SD) & $6.4(5.1)$ & $0.14(0.19)$ & .101 \\
UPC, mean (SD) & $0.39(0.22)$ & $3.54(0.70)$ & .869 \\
COC, mean (SD) & $0.12(0.16)$ & $<.001$ \\
SECON, mean (SD) & $0.15(0.19)$ & $3.49(0.68)$ & \\
VE, mean (SD) & &
\end{tabular}

Abbreviations: COC, dispersion continuity of care; SECON, sequence continuity; UPC, usual provider continuity; VE, visit entropy. * $P$-values are Fishers exact test for two valued nominal variables, Chi-Square test for multiple valued nominal variables, and $W$ ilcoxon rank sum for continuous variables.

Table 3 shows descriptive statistics of the cohort and compares the 2 groups based on the dependent variable, readmission, or death within 30 days of discharge. Patients who were readmitted within 30 days were older, had a higher Charlson score, stayed in the hospital longer, and had more previous hospitalizations and ED visits. They made more outpatient visits (mean: 12.4 vs $9.8 ; P<.001$ ) and saw more unique health care providers (mean: 7.7 vs $6.4 ; P<.001$ ). Bivariate analysis showed their continuity of care was slightly lower as measured by UPC $(0.37$ vs $0.39 ; P<.001)$ but COC $(0.12$ vs $0.12 ; P=.101)$ and SECON (0.14 vs 0.15 ; $P=.869)$ were not statistically different. VE was higher (3.54 vs $3.49 ; P<.001)$ indicating a more disorganized visit pattern and lower continuity of care. Figure 2 shows box plots of the difference in means for UPC, COC, SECON, and VE for patients readmitted versus those not readmitted. Histograms of each continuity measure are also shown below each box plot, highlighting the non-normal distribution of UPC, COC, and SECON.

The 4 multivariate models demonstrate that once known readmission risk factors were controlled, continuity of care as measured by UPC, COC, and SECON was not significantly associated with readmission. However, VE remained a significant factor with an odds ratio of $1.10(95 \%$ confidence interval, 1.02 to 1.19 ). Figure 1 shows the pooled odds ratios for each independent variable in the 4 models along with $95 \%$ confidence intervals. The forest plot also graphically shows the odds ratio $95 \%$ confidence intervals for UPC, COC, and SECON include 1.0, while VE does not.

\section{Discussion}

Patients with higher VE in the 12 months before hospital admission were more likely to be readmitted or die within 30 days of hospital discharge. This effect was independent of medical comorbidities or utilization factors as measured by the Charlson Comorbidity Index, the number of prior ED visits, and the number of prior hospitalizations in the multivariate analysis. Higher VE reflects a more disorganized visit pattern and a lack of continuity of care. Thus, decreased continuity of care before admission is associated with increased odds of readmission in our study. A single unit change in VE, equivalent to visiting a single physician 4 times $(\mathrm{VE}=1.31$; Table 2, Case $\mathrm{H})$ versus visiting 4 different physicians once each $(\mathrm{VE}=2.37$; Table 2, Case $\mathrm{C}$ ), produces approximately the same change 
Figure 1. Multivariate odds ratio forest plot for independent variables and continuity measures associated with 30-day readmission. CI, confidence interval; ED, emergency department; UPC, usual provider continuity; COC, dispersion continuity of care; SECON, sequence continuity; VE, visit entropy.

Variable

Age

Gender (Male)

Marital Status (Married)

Charlson Score

\# ED Visits, $6 \mathrm{mo}$

\# Admissions, 12mo

Length of Stay

Continuity Measures

UPC

$\operatorname{coc}$

SECON

VE

$\begin{array}{rr}\text { Odds Ratio } & 95 \% \text { Cl } \\ 1.000 & (0.997-1.003) \\ 0.998 & (0.893-1.116) \\ 0.903 & (0.806-1.011) \\ 1.102 & (1.084-1.120) \\ 1.069 & (1.039-1.100) \\ 1.159 & (1.083-1.239) \\ 1.062 & (1.049-1.075) \\ & \\ 0.880 & (0.672-1.145) \\ 0.693 & (0.466-1.012) \\ 0.826 & (0.604-1.120) \\ 1.103 & (1.020-1.191)\end{array}$

in odds of readmission as an additional Charlson Comorbidity Index point. In other words, reduced continuity is as important a factor in hospital readmissions as the history of myocardial infarction, congestive heart failure, chronic obstructive pulmonary disease, or diabetes without end-organ damage.

Team-based models of care increase collaboration between primary care physicians, subspecialists, midlevel providers, care managers, nurses, dietitians, pharmacists, physical therapists, social workers, and psychologists. This comprehensive patient management by a limited set of health care providers who are familiar with the patient's "story" forms the foundation of a PCMH and may increase quality of care. ${ }^{11} \mathrm{~A}$ high-functioning care team helps to support a patient's transition of care from hospital to home, something that is essential in reducing readmissions. ${ }^{44} \mathrm{VE}$ essentially measures the organization of care. Thus, it might prove useful in defining patients at risk for readmission due to a lack of organized care. Of course, further prospective studies are necessary to determine whether actively improving a patient's VE reduces readmission rates.

In the multivariate model, the other measures of continuity (UPC, COC, and SECON) did not show this same association. We believe that this is due to technical limitations of these measures. As shown in Figure 2, the distributions of these measures are highly skewed whereas VE has an approximately normal distribution. Having zero or 1 outpatient visit, which occurs in upwards of $50 \%$ of all primary care patients over a 1 -year period, ${ }^{45}$ results in division by zero as demonstrated by the formulas in Table 1 for UPC, COC, and SECON. Division by zero is mathematically undefined, thus excluding significant numbers of patients using these measures $\left(473,1242\right.$, and 1242 respectively). ${ }^{27,46} \mathrm{In}$ contrast, $\mathrm{VE}$ is defined even if the patient makes no outpatient visits during the study period. Furthermore, $\mathrm{VE}$ can distinguish planned care referrals to a limited set of subspecialists (Table 2, case F) from an equivalent number of unplanned visits to random providers (Table 2, cases $\mathrm{C}$ and $\mathrm{D}$ ), thus detecting organization of care typically seen in PCMHs. ${ }^{27}$ 
Figure 2. Prior 12-month continuity measures versus 30-day readmission.

Usual Provider Continuity (UPC)
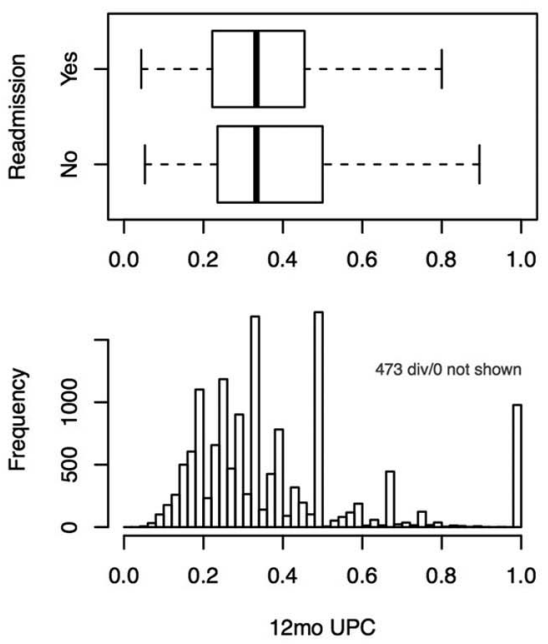

Sequence Continuity (SECON)
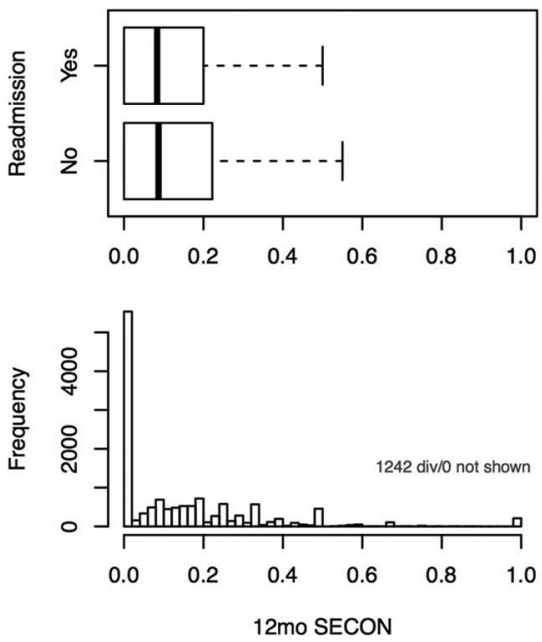

The multivariate analysis confirms many previous studies showing age, comorbidities, length of stay, and number of previous hospitalizations or ED visits are associated with increased odds of readmission. ${ }^{35-43}$ Marital status, which may be a marker of social determinants of health, and gender have had variable associations with readmission. ${ }^{41,42,47}$ In this study, neither gender nor marital status was associated with readmission odds.

\section{Limitations}

Our study was conducted at a single academic medical institution with 5 primary care clinical sites that are PCMHs. Individual variation between patient's visit patterns within our PCMH produced the dif-
Dispersion Continuity of Care (COC)
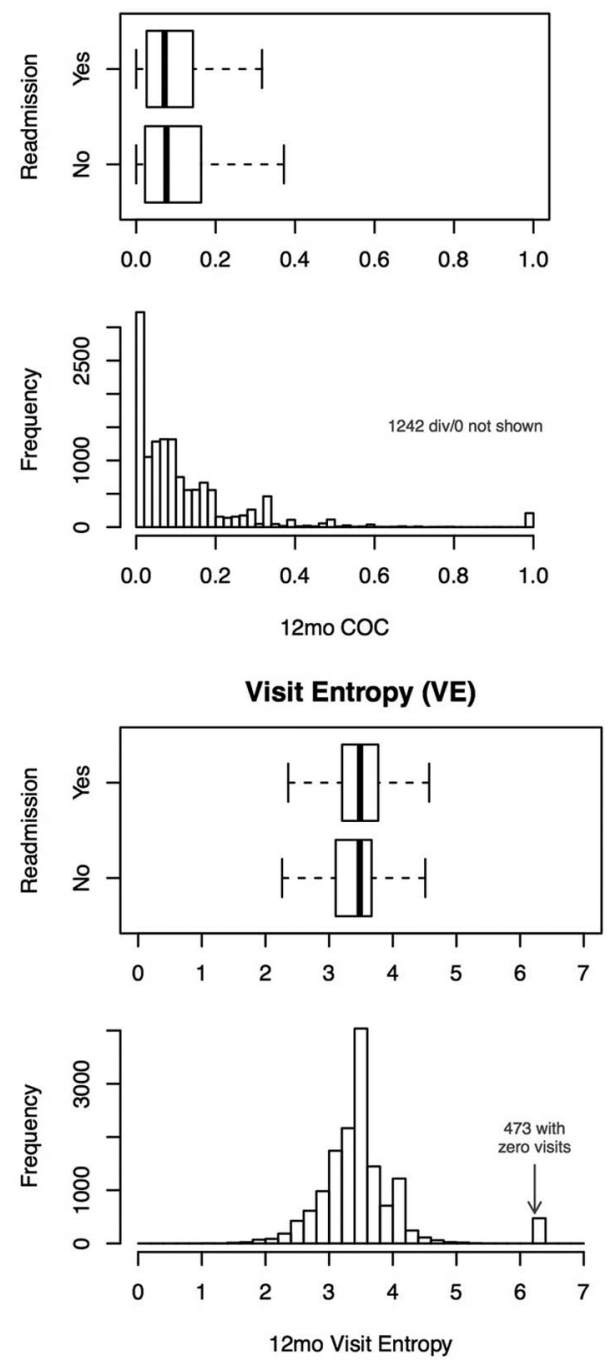

ferences we observed. We did not compare a PCMH to usual care, nor did we prospectively seek to improve visit organization and continuity within the PCMH. Further study is necessary to determine whether these findings are generalizable to other primary care practices, especially communitybased practices. We did not examine cost data; thus, further work is indicated to determine whether organized care that reduces remissions is less costly.

In addition, we were unable to quantify visits to out-of-network clinics and hospitals. Out-of-network outpatient visits decrease continuity. Based on the practice experience of the investigators, the out-of-network visits among our patients are mostly ED visits, a factor known to be a significant predictor of readmission. ${ }^{41}$ Our inability to quan- 
tify out-of-network outpatient visits tends to elevate measured continuity in a group more likely to be readmitted, thus perhaps understating the relationship between lack of continuity and readmission that we observed. Furthermore, many of our patients have insurance plans that actively discourage use of out-of-network providers and thus outof-network hospitalizations are rare.

The exclusion of frail high-readmission-risk elders enrolled in a care transitions program may attenuate the observed relationship between age, Charlson score, acute medical service utilization, and the odds of readmission. This patient group's $\mathrm{VE}$ would be low due to the highly integrated nature of this program. Regardless, any impact on our results should be minimal given the small number of patients involved. Finally, the inclusion of planned readmissions in the analysis may attenuate the relationship between $\mathrm{VE}$ and odds of readmission since those with planned readmissions likely have more organized care and thus a lower VE.

\section{Conclusion}

Lower continuity of care, characterized by more disorganized visit pattern and thus higher $\mathrm{VE}$, is associated with increased odds of readmission as seen in Figure 1. An association between traditional measures of continuity (UPC, COC, and SECON) and readmission was not found, perhaps because these traditional measures suffer from skewed distributions, undefined division by zero, and the inability to distinguish planned team-based care from random visits. Organized medical care, a key principle of the chronic disease model and PCMHs, is associated with lower odds of readmission among hospitalized primary care patients.

We thank Julie Maxson for her invaluable assistance in collecting the data that made this study possible. Funding for this study was provided by Mayo CCaTS Grant UL1TR000135.

To see this article online, please go to: http://jabfm.org/content/ 30/1/63.full.

\section{References}

1. Jencks SF, Williams MV, Coleman EA. Rehospitalizations among patients in the Medicare fee-for-service program. N Engl J Med 2009;360:1418-28.

2. van Walraven C, Bennett C, Jennings A, Austin PC, Forster AJ. Proportion of hospital readmissions deemed avoidable: A systematic review. CMAJ 2011; 183:E391-E402.
3. Ashton CM, Kuykendall DH, Johnson ML, Wray $\mathrm{NP}, \mathrm{Wu} \mathrm{L}$. The association between the quality of inpatient care and early readmission. Ann Intern Med 1995;122:415-21.

4. Balla U, Malnick S, Schattner A. Early readmissions to the department of medicine as a screening tool for monitoring quality of care problems. Medicine (Baltimore) 2008;87:294-300.

5. Halfon P, Eggli Y, Prêtre-Rohrbach I, Meylan D, Marazzi A, Burnand B. Validation of the potentially avoidable hospital readmission rate as a routine indicator of the quality of hospital care. Med Care 2006;44:972-81.

6. Balaban RB, Weissman JS, Samuel PA, Woolhandler S. Redefining and redesigning hospital discharge to enhance patient care: A randomized controlled study. J Gen Intern Med 2008;23:1228-33.

7. Coleman EA, Parry C, Chalmers S, Min SJ. The care transitions intervention: Results of a randomized controlled trial. Arch Intern Med 2006;166: 1822-8.

8. Naylor MD, Brooten D, Campbell R, et al. Comprehensive discharge planning and home follow-up of hospitalized elders: A randomized clinical trial. JAMA 1999;281:613-20.

9. Dudas V, Bookwalter T, Kerr KM, Pantilat SZ. The impact of follow-up telephone calls to patients after hospitalization. Am J Med 2001;111:26S-30S.

10. Phillips CO, Wright SM, Kern DE, Singa RM, Shepperd S, Rubin HR. Comprehensive discharge planning with postdischarge support for older patients with congestive heart failure: a meta-analysis. JAMA 2004;291:1358-67.

11. Rosenthal TC. The medical home: Growing evidence to support a new approach to primary care. J Am Board Fam Med 2008;21:427-40.

12. Donaldson MS, Yordy KD, Lohr KN, Vanselow NA. Primary Care: America's Health in a New Era. Washington, DC: The National Academies Press; 1996.

13. Bodenheimer T, Wagner EH, Grumbach K. Improving primary care for patients with chronic illness. JAMA 2002;288:1775-9.

14. Ferrante JM, Balasubramanian BA, Hudson SV, Crabtree BF. Principles of the patient-centered medical home and preventive services delivery. Ann Fam Med 2010;8:108-16.

15. Saultz JW, Lochner J. Interpersonal continuity of care and care outcomes: A critical review. Ann Fam Med 2005;3:159-66.

16. Cabana MD, Jee SH. Does continuity of care improve patient outcomes? J Fam Pract 2004;53:974-80.

17. Smith CS. The impact of an ambulatory firm system on quality and continuity of care. Med Care 1995; 33:221-6.

18. Weinberger M, Oddone EZ, Henderson WG. Does increased access to primary care reduce hospital readmissions? Veterans Affairs Cooperative Study 
Group on Primary Care and Hospital Readmission. N Engl J Med 1996;334:1441-7.

19. Mainous AG 3rd, Gill JM. The importance of continuity of care in the likelihood of future hospitalization: Is site of care equivalent to a primary clinician? Am J Public Health 1998;88:1539-41.

20. Gill JM. Can hospitalizations be avoided by having a regular source of care? Fam Med 1997;29:166-71.

21. Menec VH, Sirski M, Attawar D, Katz A. Does continuity of care with a family physician reduce hospitalizations among older adults? J Health Serv Res Policy 2006;11:196-201.

22. Cheng SH, Chen CC, Hou YF. A longitudinal examination of continuity of care and avoidable hospitalization: Evidence from a universal coverage health care system. Arch Intern Med 2010;170: 1671-7.

23. Jee SH, Cabana MD. Indices for continuity of care: A systematic review of the literature. Med Care Res Rev 2006;63:158-88.

24. Breslau N, Reeb KG. Continuity of care in a university-based practice. J Med Educ 1975;50:965-9.

25. Eriksson EA, Mattsson LG. Quantitative measurement of continuity of care. Measures in use and an alternative approach. Med Care 1983;21:858-875.

26. Rodriguez HP, Rogers WH, Marshall RE, Safran DG. The effects of primary care physician visit continuity on patients' experiences with care. J Gen Intern Med 2007;22:787-93.

27. Garrison GM, Bania B. Visit entropy: Comparing a novel method to existing continuity of care measures. Eur J Pers Cent Healthc 2015;3:343-51.

28. Shannon CE. A mathematical theory of communication. Bell Syst Tech J 1998;15:57-64.

29. Cover TM, Thomas JA. Elements of information theory. 2nd Ed. Hoboken, New Jersey: John Wiley \& Sons, Inc; 2006.

30. Rogers J, Curtis P. The concept and measurement of continuity in primary care. Am J Public Health 1980; $70: 122-7$.

31. Mattsson LG, Westman G. Evaluation of provider continuity in primary care: Actual versus random and potential continuity. Fam Pract 1987;4:251-59.

32. Saultz JW. Defining and measuring interpersonal continuity of care. Ann Fam Med 2003;1:134-43.

33. Charlson ME, Pompei P, Ales KL, MacKenzie CR. A new method of classifying prognostic comorbidity in longitudinal studies: Development and validation. J Chronic Dis 1987;40:373-83.

34. Halfon P, Eggli Y, van Melle G, Chevalier J, Wasserfallen JB, Burnand B. Measuring potentially avoidable hospital readmissions. J Clin Epidemiol 2002;55:573-87.
35. Marcantonio ER, McKean S, Goldfinger M, Kleefield S, Yurkofsky M, Brennan TA. Factors associated with unplanned hospital readmission among patients 65 years of age and older in a Medicare managed care plan. Am J Med 1999;107:13-7.

36. Billings J, Dixon J, Mijanovich T, Wennberg D. Case finding for patients at risk of readmission to hospital: development of algorithm to identify high risk patients. BMJ 2006;333:327-8.

37. Arbaje AI, Wolff JL, Yu Q, Powe NR, Anderson GF, Boult C. Postdischarge environmental and socioeconomic factors and the likelihood of early hospital readmission among community-dwelling Medicare beneficiaries. Gerontologist 2008;48:495-504.

38. Silverstein MD, Qin H, Mercer SQ, Fong J, Haydar Z. Risk factors for 30-day hospital readmission in patients $\geq 65$ years of age. Proc (Bayl Univ Med Cent) 2008;21:363-72.

39. Hasan O, Meltzer DO, Shaykevich SA, et al. Hospital readmission in general medicine patients: A prediction model. J Gen Intern Med 2010;25:211-9.

40. Billings J, Blunt I, Steventon A, Georghiou T, Lewis G, Bardsley M. Development of a predictive model to identify inpatients at risk of re-admission within 30 days of discharge (PARR-30). BMJ Open 2012;2: e001667.

41. Garrison GM, Mansukhani MP, Bohn B. Predictors of thirty-day readmission among hospitalized family medicine patients. J Am Board Fam Med 2013;26: 71-7.

42. Shulan M, Gao K, Moore CD. Predicting 30-day all-cause hospital readmissions. Health Care Manag Sci 2013;16:167-75.

43. Shadmi E, Flaks-Manov N, Hoshen M, Goldman O, Bitterman H, Balicer RD. Predicting 30-day readmissions with preadmission electronic health record data. Med Care 2015;53:283-9.

44. Misky GJ, Wald HL, Coleman EA. Post-hospitalization transitions: Examining the effects of timing of primary care provider follow-up. J Hosp Med 2010;5:392-7.

45. Garrison GM, Traverse CR, Fish RG. A case study of visit-driven preventive care screening using clinical decision support: The need to redesign preventive care screening. Health Serv Res Manag Epidemiol 2016; doi:10.1177/2333392816650344.

46. Rohrer JE, Angstman KB, Garrison GM, Maxson JA, Furst JW. Family medicine patients who use retail clinics have lower continuity of care. J Prim Care Community Health 2013;4:150-3.

47. Kansagara D, Englander H, Salanitro A, et al. Risk prediction models for hospital readmission: A systematic review. JAMA 2011;306:1688-98. 